\title{
La importancia de utilizar herramientas y soportes de la comunicación en el proceso enseñanza-aprendizaje en el Instituto Tecnológico de Veracruz
}

Importance of Using Communication Tools and Supports in the TeachingLearning Process in the Instituto Tecnológico de Veracruz

A importância do uso de ferramentas e suportes de comunicação no processo de ensino-aprendizagem do Instituto Tecnológico de Veracruz

Marina Cecilia Pérez Castillo

Tecnológico Nacional de México, Instituto Tecnológico de Veracruz, México marina.pc@veracruz.tecnm.mx https://orcid.org/0000-0002-6302-7978

Perfecto Gabriel Trujillo Castro

Tecnológico Nacional de México, Instituto Tecnológico de Veracruz, México perfecto.tc@veracruz.tecnm.mx https://orcid.org/0000-0001-7511-7674

Sonia Báez Lagunes

Tecnológico Nacional de México, Instituto Tecnológico de Veracruz, México sonia.bl@veracruz.tecnm.mx https://orcid.org/0000-0001-6429-9388

Bertha Conde Carreño

Tecnológico Nacional de México, Instituto Tecnológico de Veracruz, México bertha.cc@veracruz.tecnm.mx https://orcid.org/0000-0002-7470-628X 


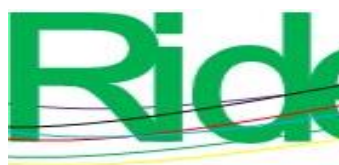

Revista Iberoamericana para la
Investigación y el Desarrollo Educativo
ISSN $2007-7467$

Selene Magdala Ramos Cortés

Tecnológico Nacional de México, Instituto Tecnológico de Veracruz, México

selene.rc@veracruz.tecnm.mx

https://orcid.org/0000-0001-5689-4224

\section{Resumen}

La incorporación de las herramientas y soportes de la comunicación en el proceso de enseñanza-aprendizaje da la posibilidad de acceder a una gran cantidad de información. Y también ofrece la oportunidad de que el profesor abandone su actividad tradicional consistente en la transmisión de conocimientos y comience a dirigir sus esfuerzos hacia el aprendizaje del alumnado. La docencia, por lo tanto, se dirige hacia el desarrollo de procesos de aprendizaje con la finalidad de orientar al estudiante hacia la creación de su propio conocimiento a partir del conjunto de recursos de información disponible. De allí la necesidad imperiosa de que tanto en el aula como en el laboratorio y en el entorno del profesor se haga uso de ambos. El objetivo de la presente investigación es determinar el conocimiento y el uso de las herramientas y soportes de la comunicación de los docentes y estudiantes en la licenciatura en Administración e ingeniería en Gestión Empresarial que se imparten en el Instituto Tecnológico de Veracruz, dependiente del Tecnológico Nacional de México.

Palabras clave: comunicación, educación, herramientas, soportes.

\section{Abstract}

The incorporation of communication tools and supports in the teaching-learning process gives the possibility of accessing a large amount of information. And also provides the opportunity for the teacher to abandon their traditional activity consisting in the transmission of knowledge and begin to direct their efforts towards student learning. Teaching, therefore, is directed towards the development of learning processes in order to guide the student towards the creation of their own knowledge from the set of available information resources. That is why the imperative need for them to be used both in the classroom, laboratory and in the teacher's environment. The objective of this research is to determine the knowledge and use of communication tools and supports for teachers and students in the degree and 

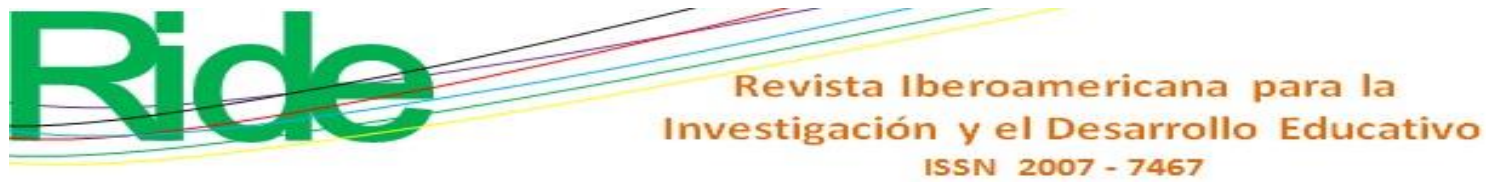

engineering in Business Administration in the Instituto Tecnológico de Veracruz, dependent of the Tecnológico Nacional de México.

Keywords: communication, education, tools, support.

\section{Resumo}

A incorporação de ferramentas e suportes de comunicação no processo de ensinoaprendizagem oferece a possibilidade de acessar uma grande quantidade de informações. E também oferece a oportunidade para o professor abandonar sua atividade tradicional que consiste na transmissão de conhecimento e começar a direcionar seus esforços para a aprendizagem do aluno. O ensino, portanto, é direcionado ao desenvolvimento de processos de aprendizagem, a fim de orientar o aluno para a criação de seu próprio conhecimento a partir do conjunto de recursos de informação disponíveis. Daí a necessidade urgente de que, tanto na sala de aula como no laboratório e no ambiente do professor, se utilizem de ambos. O objetivo desta pesquisa é determinar o conhecimento e o uso de ferramentas e suportes de comunicação para professores e alunos do Bacharelado em Administração de Empresas e Engenharia ministrado no Instituto Tecnológico de Veracruz, dependente do Instituto Tecnológico Nacional. do México.

Palavras-chave: comunicação, educação, ferramentas, suportes.

Fecha Recepción: Septiembre 2019

Fecha Aceptación: Junio 2020

\section{Introducción}

La historia de los soportes y herramientas de la comunicación en el Instituto Tecnológico de Veracruz ha sido la siguiente: a inicios de los 70 existió una computadora IBM 1130 que todavía utilizaba tarjetas para perforar y correr los programas, utilizada para las actividades administrativas y por las áreas académicas. En 1992 se instaló la primera PC en el área administrativa y posteriormente se introdujeron computadoras para los laboratorios de las ingenierías; las carreras de ingeniería en Sistemas Computacionales y Electrónica tuvieron prioridad. En el año 2005 se inaugura el Laboratorio de Informática Administrativa para uso de los alumnos de la licenciatura en Administración, y en el año 2010 se implementa el Simulador de Negocios, que es utilizado por los alumnos de las licenciatura en 


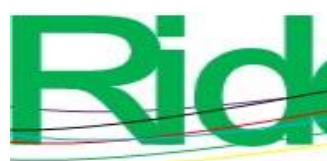

Revista Iberoamericana para la
Investigación y el Desarrollo Educativo ISSN $2007-7467$

Administración e ingeniería en Gestión Empresarial para actividades académicas. Actualmente se cuenta con una red de internet que comunica a todos los usuarios en el plantel: alumnos, docentes y administrativos.

\section{Justificación}

La presente investigación es necesaria debido a que el uso de los soportes y herramientas de la comunicación en la licenciatura en Administración y en la ingeniería en Gestión Empresarial beneficia nuevos estilos de aprendizaje y educación, al facilitar la comunicación desde cualquier lugar y en cualquier momento y al dar mayor autonomía, por mencionar tan solo un par características que han convertido a estas herramientas en unas de las más importantes en la educación formal e informal.

\section{Marco teórico}

De acuerdo con Muñoz, Rodríguez y Barrera (2013), “el mejoramiento y eficiencia de los centros educativos es una de las principales preocupaciones de teóricos y prácticos en educación desde hace varias décadas" (p. 100).

Para Chase y Aquilano (1995, citados en Silva, Cruz, Méndez y Hernández, 2013), las instituciones de educación superior "no solo deben ser eficaces, sino que deben buscar y alcanzar la eficiencia. La eficacia es la obtención de los resultados deseados y la eficiencia se logra cuando se obtiene un resultado deseado con el mínimo de insumos” (p. 105).

Un docente innovador es el que busca capacitarse, el que investiga y participa en esta nueva sociedad del conocimiento. Es también un docente que se adapta al aprendizaje permanente y busca estrategias para adquirir habilidades en el uso de programas y aplicaciones que sirvan para innovar en sus clases.

Toda institución de educación superior debe planificar la integración de las herramientas y soportes de la comunicación, haciendo participar a todos los involucrados, tales como: funcionarios, docentes y alumnos. Y es aquí donde la gestión educativa y el uso de las herramientas y soportes de la comunicación implica, a su vez, la gestión de procesos claves que contribuyan a la toma de decisiones estratégicas, basándose en el modelado de procesos, de preferencia que sea un proceso automatizado. 


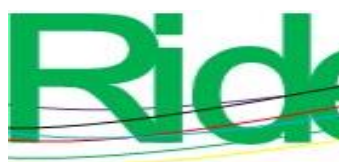

Revista Iberoamericana para la
Investigación y el Desarrollo Educativo
ISSN $2007-7467$

Por un lado, maestros y alumnos necesitan incorporar a sus quehaceres las habilidades y destrezas en el manejo de la tecnología educativa y, por el otro, requieren no solo de estrategias educativas apropiadas para la potenciación del aprendizaje, sino, por supuesto, de las herramientas para lograr el objetivo del quehacer docente. Esto es: las herramientas y soportes de la comunicación. También, valga decir, se requiere el ejercicio de la inteligencia emocional.

Alfie y Veloso (2011) explican lo siguiente:

En el siglo XXI, el docente se encuentra en el contexto de la sociedad del conocimiento, en la cual las nuevas tecnologías como Internet, con la super abundante producción de información, han generado cambios en todos los ámbitos. Los docentes también deben ser protagonistas de estos cambios para pasar de simples espectadores a transformadores de la sociedad, incorporando el "aprendizaje permanente y durante toda la vida" (p. 7).

También afirman lo puesto a continuación:

El docente debe contar con las competencias de estar capacitado en las herramientas y soportes de la comunicación necesarias para favorecer en sus estudiantes la adquisición de habilidades imprescindibles para llegar a ser: competentes para utilizar las tecnologías, buscadores, analizadores $\mathrm{y}$ evaluadores de información, solucionadores de problemas y tomadores de decisiones, usuarios creativos y eficaces en el manejo de las herramientas de productividad, comunicadores, colaboradores, publicadores, y productores, ciudadanos informados, responsables y capaces de contribuir a la sociedad (Alfie y Veloso, 2011, p. 7).

Orientado por el docente, el estudiante conoce las técnicas de enseñanza, y amplía su visión a través de las estrategias de aprendizaje, gracias a lo cual tendría la oportunidad de lograr su competencia, siempre en relación con su experiencia, conocimientos anteriores, aptitudes y actitudes. 


\section{Metodología}

Se consideraron las poblaciones de dos de las licenciaturas del Instituto Tecnológico de Veracruz, perteneciente al Tecnológico Nacional de México, a saber, la licenciatura en Administración con una población de 379 alumnos y la ingeniería en Gestión Empresarial con una población de 264 alumnos. Se utilizó un método para cálculo de muestras grandes $\geq 30$.

El cálculo de las muestras de cada una de ellas es la siguiente: se utiliza un muestreo estratificado dado que la población de alumnos del Departamento Académico de Ciencias Económico-Administrativas es de 643. Para el muestreo, esta población se ha dividido en las dos licenciaturas que atiende el dicho departamento, como se mencionó anteriormente, y en la edad de los alumnos que oscila entre 16 y 35 años.

Para los docentes se buscó aplicar un censo al total de estos, que son 60.

- Muestra para la licenciatura en Administración:

$$
\begin{gathered}
\mathrm{N}=\frac{\mathrm{Z}^{2} \mathrm{Npq}}{\mathrm{NE}^{2}+\mathrm{Z}^{2} \mathrm{pq}}=\frac{(1.96)^{2}(379)(0.5)(0.5)}{(379)(0.05)^{2}+(1.96)^{2}(0.5)(0.5)} \\
\mathrm{N}=\frac{363.9916}{0.9475+0.9604}=\frac{363.9916}{1.6204}=190.78=191 \text { encuestas }
\end{gathered}
$$

- Muestra para la ingeniería en Gestión Empresarial:

$$
\begin{gathered}
\mathrm{N}=\frac{\mathrm{Z}^{2} \mathrm{Npq}}{\mathrm{NE}^{2}+\mathrm{Z}^{2} \mathrm{pq}}=\frac{(1.96)^{2}(264)(0.5)(0.5)}{(264)(0.05)^{2}+(1.96)^{2}(0.5)(0.5)} \\
\mathrm{N}=\frac{129.36}{0.66+0.9604}=\frac{129.36}{1.6204}=79.8321=80 \text { encuestas }
\end{gathered}
$$

Se utilizaron y aplicaron encuestas de Internet: para docentes se recurrió al sitio www.e-encuesta.com y la encuesta para alumnos se tomó de la Universitat Oberta de Catalunya, con dirección electrónica de http://ijedict.uwi.edu/include/getdoc.php?id01161\&pubkic=true. En ambos casos, la encuesta fue adaptada de acuerdo con los fines de esta investigación. 
Resultados

Los resultados de las encuestas aplicadas se exponen a continuación.

\section{Resultados de encuestas aplicadas a los docentes}

En este apartado se muestran los resultados de las encuestas aplicadas a los docentes del Departamento de Ciencias Económico-Administrativas, que tiene a su cargo las carreras de la licenciatura en Administración e ingeniería en Gestión Empresarial, del Tecnológico Nacional de México, Instituto Tecnológico de Veracruz, México. La encuesta fue aplicada a 60 docentes: contestaron 54, cifra que representa $90 \%$.

Se presentan a continuación los resultados de los rubros estudiados.

\section{Comunicación}

En primer lugar, $87 \%$ de los encuestados sí conoce las herramientas y soportes de la comunicación y $13 \%$ no. Asimismo, $43 \%$ considera que utiliza entre $50 \%$ y $65 \%$ las herramientas y soportes de la comunicación en su clase. Y $93 \%$ de los encuestados cree que los recursos tecnológicos favorecen la adquisición de aprendizajes gracias a los ambientes de las diversas comunidades virtuales; el restante $7 \%$ no cree en esto.

Los resultados que arroja la encuesta aplicada demuestran que un porcentaje significativo aunque no mayoritario de docentes utiliza las nuevas tecnologías mediante dispositivos móviles para comunicarse con sus alumnos. Dicha información se presenta de manera gráfica en la figura 1 . 

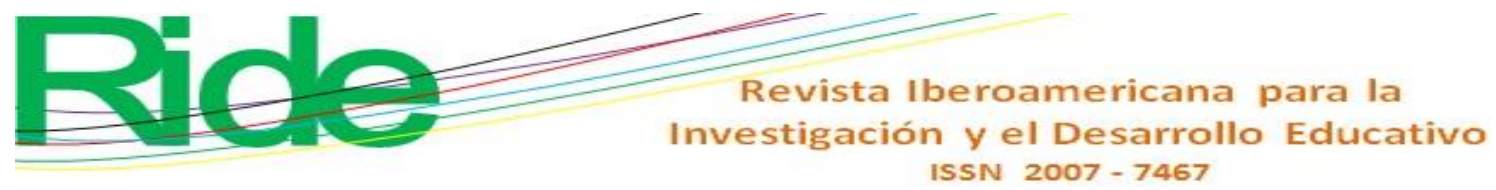

Figura 1. Docentes que utilizan las nuevas tecnologías mediante dispositivos móviles para comunicarse con sus alumnos



Fuente: Elaboración propia

\section{Procesos de enseñanza}

En este rubro, $87 \%$ de los encuestados menciona que es necesario la importancia de la utilización de recursos tecnológicos como apoyo didáctico en los procesos de enseñanza y $13 \%$ lo considera opcional.

Los resultados obtenidos en la investigación permiten destacar que los docentes consideran la falta de disponibilidad de equipos y materiales en el uso de herramientas y soportes de la comunicación como una desventaja para su actividad docente, tal como se presenta en la figura 2.

Figura 2. Docentes consideran desventaja la falta de disponibilidad de equipos y materiales en el uso de herramientas y soportes de la comunicación

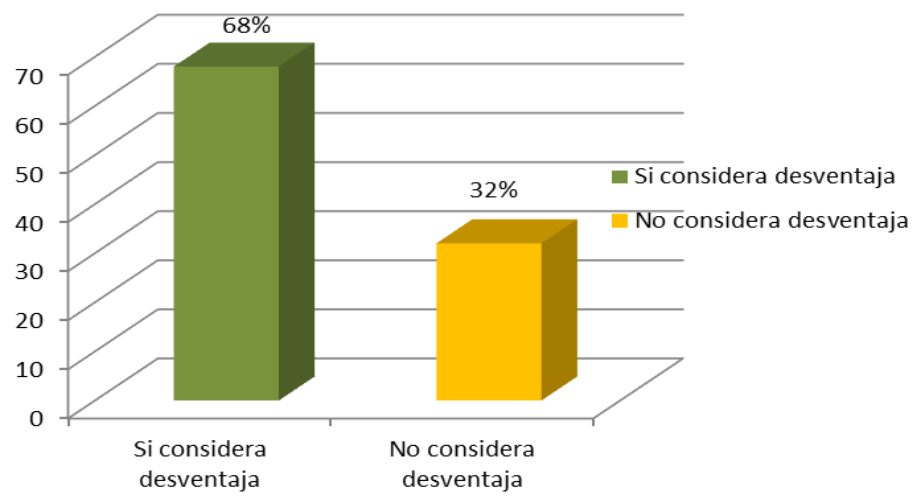

Fuente: Elaboración propia 

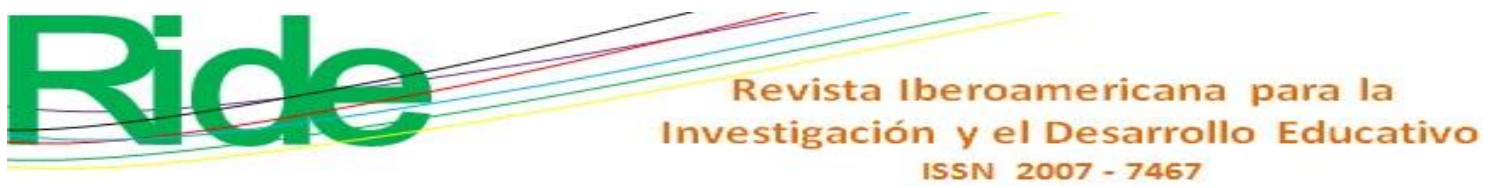

Ahora bien, $74 \%$ siente de excelente a buena confianza al momento de emplear los medios tecnológicos frente a grupo.

Asimismo, $35 \%$ considera que el uso de las herramientas y soportes de la comunicación en clase son una herramienta de apoyo, una alternativa para la enseñanza de los diversos contenidos.

Sin embargo, como se ha visto en la figura 2, $68 \%$ de los encuestados considera una desventaja la falta de disponibilidad de equipos y materiales en el uso de las herramientas y soportes de la comunicación en el salón de clase.

Por otro lado, a $59 \%$ de los docentes le gustaría conocer y aprender de sitios web relacionados con las nuevas herramientas y soportes de la comunicación; $66 \%$ de los docentes le gustaría conocer y aprender el uso de plataformas educativas de las nuevas herramientas y soportes de la comunicación, y 67 \% le gustaría conocer y aprender el uso de software específico de las nuevas herramientas y soportes de la comunicación.

Siguiendo con la investigación, esta permite observar que los docentes consideran necesario recibir cursos especiales, como lo muestra la figura 3.

Figura 3. Docentes consideran necesario cursos especiales de formación en el uso de herramientas y soportes de la comunicación

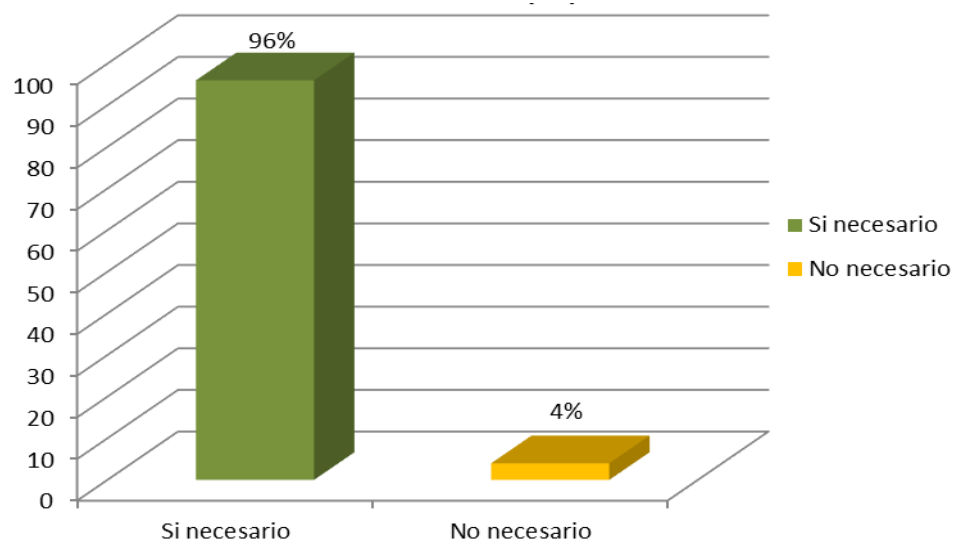

Fuente: Elaboración propia 


\section{Resultados de encuestas aplicadas a estudiantes \\ Comunicación}

Casi la totalidad de los encuestados (99\%) utiliza Internet (acceden a sitios web, $e$ mail y otros servicios de la Red).

En cuanto al tiempo que le dedican, $11 \%$ de los encuestados se conecta menos de tres horas a la semana (sitios web, e-mail u otros servicios de la Red), $25 \%$ se conecta entre cuatro y siete horas a la semana, otro $25 \%$ se conecta entre 8 y 12 horas a la semana, $21 \%$ pasa entre 13 y 20 horas navegando semanalmente y, por último, $18 \%$ se conecta más de 20 horas a la semana.

La mayoría (66 \%) casi siempre utiliza las computadoras y otras herramientas de la comunicación cuando realiza presentaciones en clase y $34 \%$ de los encuestados algunas veces las utiliza.

Cabe señalar que $45 \%$ de los encuestados nunca ha establecido comunicación online con compañeros de clase para realizar alguna actividad académica.

De los encuestados, $66 \%$ casi siempre ha tenido la oportunidad de trabajar en equipo. Y del total, $58 \%$ de los participantes algunas veces ha tenido la oportunidad de trabajar en equipo fuera del horario de clases, con apoyo del uso de las herramientas y soportes de la comunicación.

La investigación mostró el nivel de comunicación que existe entre el docente y los estudiantes mediante el correo electrónico, el cual se muestra en la figura 4.

Figura 4. Estudiantes que han enviado un e-mail a un docente para aclarar dudas

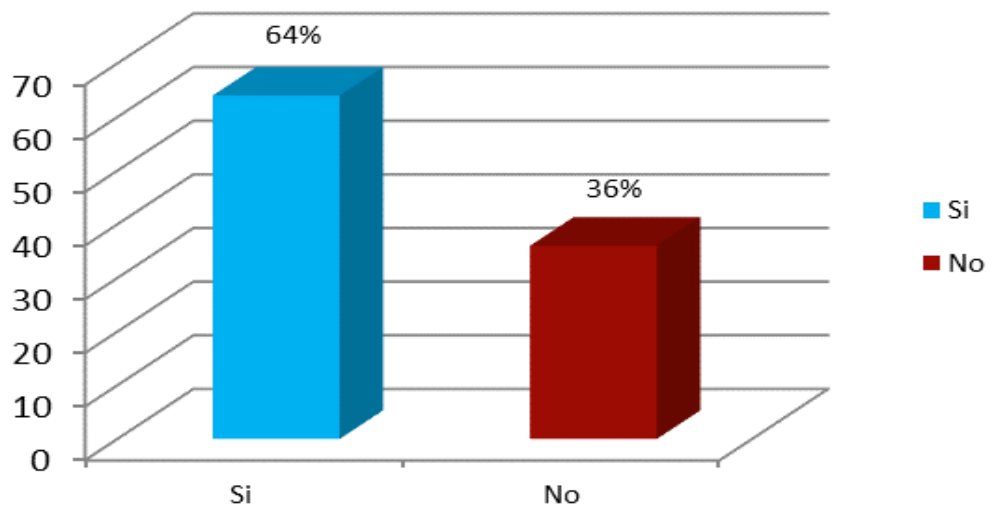

Fuente: Elaboración propia 

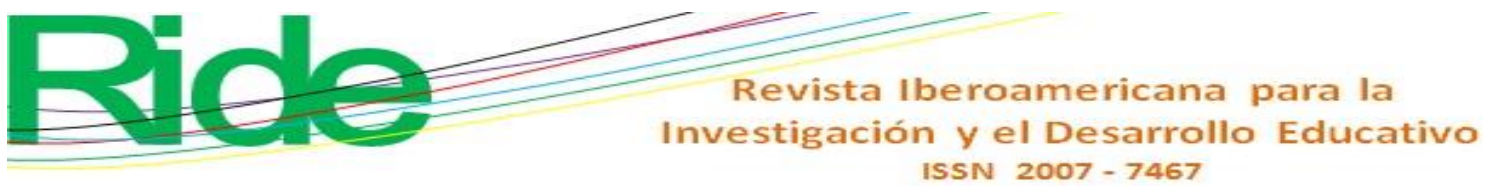

También es de resaltar que $61 \%$ de los encuestados nunca se ha dirigido a un profesor vía $e$-mail para expresarle ideas que no se atreverían a decirle cara a cara en clase.

En la figura 5 se presentan los resultados de los estudiantes que han tomado cursos online.

Figura 5. Estudiantes que no han tomado ningún curso online



Fuente: Elaboración propia

Ahondando más en el tema, $68 \%$ de los encuestados valora positivamente su habilidad en el uso de los programas informáticos, pues se creen capaces de ejecutarlos por sí solos. En esa misma línea, $90 \%$ de los encuestados considera que puede utilizar el navegador de Internet sin ayuda (ejemplo: buscar información meteorológica o bajar archivos de música).

Por último, $74 \%$ de los encuestados considera que se enfrenta con mucha confianza al uso de las herramientas y soportes de la comunicación en sus estudios universitarios.

\section{Procesos de enseñanza}

Durante su desempeño académico, $69 \%$ realiza con mayor frecuencia sus trabajos utilizando una computadora en su casa y $26 \%$ realiza con mayor frecuencia sus trabajos utilizando una computadora en la universidad.

En la figura 6 se presenta la frecuencia con que los docentes hacen requerimientos de utilizar las herramientas y soportes de la comunicación a los estudiantes. 

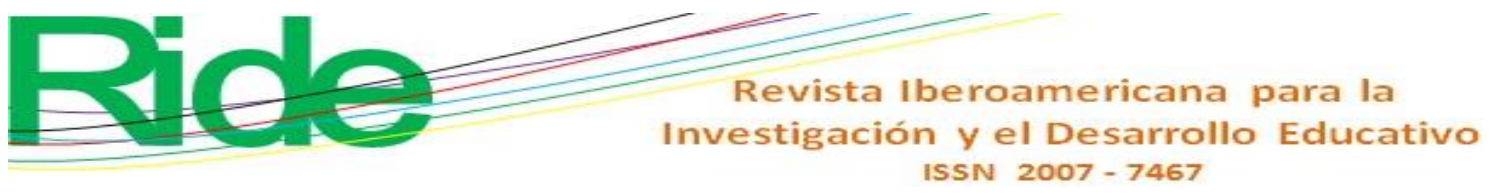

Figura 6. Docentes que piden a los estudiantes utilicen las herramientas y soportes de la comunicación

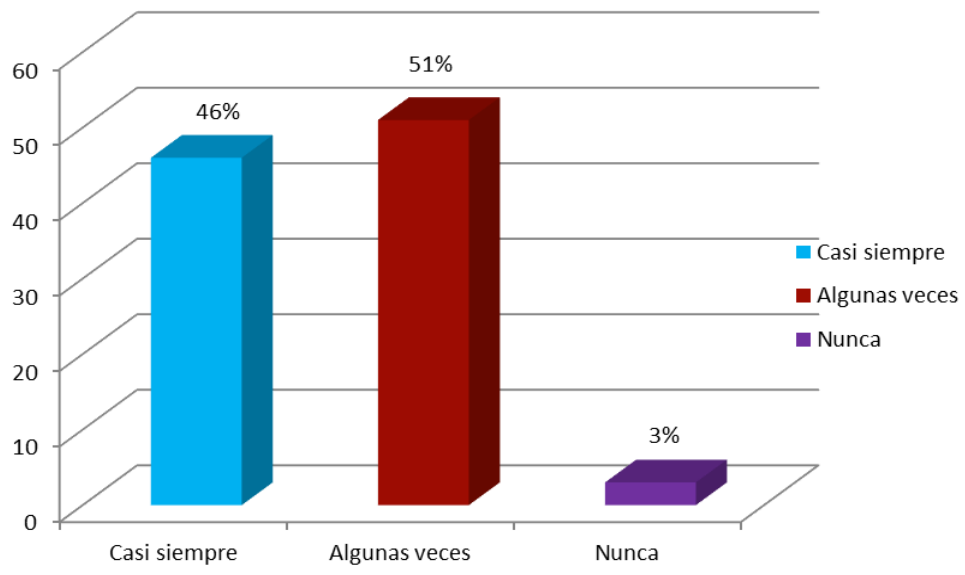

Fuente: Elaboración propia

Además, $52 \%$ de los encuestados algunas veces utiliza las páginas web de la biblioteca de su universidad para consultar el catálogo en línea, las bases de datos posibles o bajar materiales que emplea en sus actividades académicas; $38 \%$ nunca las utiliza. Mientras que $58 \%$ casi siempre recurre a la Red informática para obtener recursos que pueden emplear en sus labores académicas.

En cuanto a su capacidad para ejecutar distintas herramientas, $55 \%$ de los participantes considera que puede hacer una hoja de cálculo por sí solo (por ejemplo, utilizar Excel para hacer un documento que tenga datos numéricos simples); $90 \%$ considera que su habilidad en el uso de cualquier programa de correo electrónico es alta y no requiere de ayuda de alguien más (por ejemplo, recurrir a Outlook Express para enviar un documento o una imagen adjunta). De igual manera, $59 \%$ de los encuestados considera que su habilidad en los programas de gráficos es buena y los puede ejecutar solo (ejemplo con Paint manejar el tamaño con el color de una imagen). Incluso $48 \%$ de los alumnos considera que podría ejecutar programas de creación de páginas en Internet con un poco de ayuda (ejemplo: crear con Frontpage su propia página web). Por último, $95 \%$ de los encuestados considera que su habilidad como manejador de presentaciones es alta y puede hacerlo solo (en Power Point crear una breve presentación con diapositivas) y $88 \%$ menciona que puede consultar bases de datos bibliográficos sin supervisión, es decir, solo (ejemplo: encontrar un artículo sobre un tema de interés). 


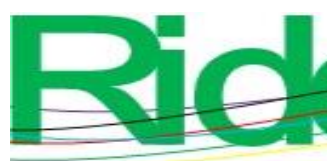

Revista Iberoamericana para la
Investigación y el Desarrollo Educativo
ISSN $2007-7467$

Ahora bien, casi el total de los participante (98\%) considera como muy importante e importante en su futura profesión las herramientas y soportes de la comunicación.

Algo negativo es que más de la mitad (67\%) casi siempre con frecuencia piensa que otros estudiantes de su universidad copian y pegan de Internet información que luego utilizan en sus trabajos sin citar la fuente.

En cuanto a las características de la población encuestada, 34\% de los encuestados son del género masculino y $66 \%$ del género femenino; $61 \%$ de los encuestados se encuentra entre los 16-20 años de edad, y $38 \%$ se encuentra entre los 21-25 años de edad. Finalmente, $62 \%$ pertenecen a la licenciatura en Administración y $28 \%$ a la ingeniería en Gestión Empresarial.

\section{Discusión}

El servidor de internet del plantel es insuficiente para todas las áreas y espacios educativos, dado que la superficie es de 20 hectáreas. El docente tiene resistencia al cambio, porque $35 \%$ considera que los soportes y herramientas de la comunicación son de uso alternativo. Los docentes argumentan falta capacitación por falta de recursos financieros.

Los estudiantes no cuentan con los equipos necesarios para acceder a Internet y a las plataformas digitales. Solo $52 \%$ de los estudiantes utiliza páginas web del plantel para sus actividades académicas.

\section{Fortalezas}

La gran mayoría de los estudiantes $(95 \%)$ puede trabajar en casa o fuera de la institución. De igual manera, 98 \% de los estudiantes considera importante para su profesión el uso de las herramientas y soportes de la comunicación. Los docentes usan las herramientas y soportes de la comunicación, y tienen disposición para emplearlas más.

\section{Debilidades}

Los docentes mencionan falta de disponibilidad de equipos y materiales en herramientas y soportes de la comunicación.

Del total de los alumnos participantes, $67 \%$ de ellos solo consulta fuentes y lo hace para copiar y pegar, sin citar las fuentes. 


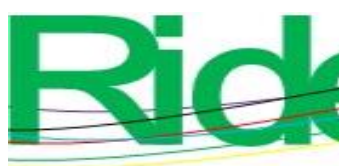

\section{Revista Iberoamericana para la Investigación y el Desarrollo Educativo ISSN 2007 - 7467}

Para futuras investigaciones se sugiere realizar el estudio en otras instituciones de educación superior para comparar los resultados.

\section{Conclusiones}

Las herramientas y soportes de la comunicación son importantes porque facilitan la comunicación desde cualquier lugar y en cualquier momento. En la presenta investigación, $78 \%$ de los docentes casi siempre hace uso de las herramientas y soportes de la comunicación. Además, $41 \%$ utiliza dispositivos móviles para comunicarse con sus alumnos.

Por otro lado, $99 \%$ de los estudiantes utilizan Internet (34 \% son hombres y $66 \%$ son mujeres). Respecto al tiempo que le dedican, $89 \%$ utiliza cuatro horas o más a la semana las herramientas y soportes de la comunicación, y $65 \%$ de ellos envía correos electrónicos a profesores para aclarar dudas sobre algún trabajo.

Sin duda estas herramientas benefician nuevos estilos de aprendizaje y educación. Al respecto, $35 \%$ de los docentes considera que las herramientas y soportes de la comunicación son un apoyo alternativo para la enseñanza; $87 \%$ sí conoce las herramientas y soportes de la comunicación. Es de destacar que a $66 \%$ de los docentes le gustaría conocer plataformas educativas; $96 \%$ considera necesario cursos especiales de formación de las herramientas y soportes de la comunicación, y $43 \%$ de los docentes usa las herramientas y soportes de la comunicación entre $50 \%$ y $65 \%$ en su clase.

Del otro lado del escritorio, $52 \%$ de los estudiantes utilizan páginas web de la biblioteca de su universidad para actividades académicas.

Aunado a todo lo anterior, lo resultados aquí obtenidos respaldan la idea de que estas herramientas permiten mayor autonomía, ya que $95 \%$ de los estudiantes usa las herramientas en la casa o en la universidad y $58 \%$ ha tenido la oportunidad de trabajar en equipo fuera de clase.

También es valioso resaltar que $87 \%$ los docentes considera que sí es necesario la utilización de recursos tecnológicos, $74 \%$ siente de excelente a buena confianza al momento de emplear medios tecnológicos frente a grupo. Los docentes mencionan en $78 \%$ la desventaja de la falta de disponibilidad de equipo y materiales en el uso de las herramientas y soportes de la comunicación. 

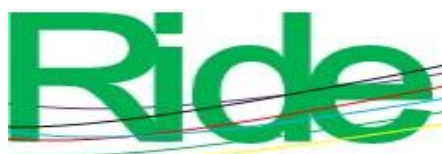

Revista lberoamericana para la
Investigación y el Desarrollo Educativo
ISSN $2007-7467$

Por otro parte, $58 \%$ del alumnado recurre a la Red para obtener recursos para las actividades académicas, y 67 \% sugiere que solo copia y pega sin citar la fuente. Más de la mitad de los estudiantes (64 \%) enfrenta con confianza el uso de las herramientas y soportes de la comunicación en sus estudios y $98 \%$ considera importante en su profesión el uso de los soportes y herramientas de la comunicación.

Es posible incrementar el uso de las herramientas y soportes de la comunicación en el futuro en el proceso de enseñanza-aprendizaje para eficientar ambientes de aprendizaje para la formación de competencias no solo pedagógicas, sino para toda la vida.

\section{Contribuciones a futuras líneas de investigación}

Como futuras líneas de investigación se propone que, a través de la capacitación de los docentes, se generen materiales didácticos en plataformas educativas que faciliten el proceso de enseñanza-aprendizaje del estudiante. Generar cursos y diplomados en línea al interior y al exterior de la institución educativa. Que los docentes se puedan certificar y convertirse en tutores en línea. Y facilitar la utilización de las plataformas virtuales para la educación a distancia. 


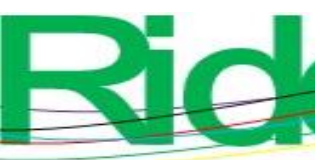

\section{Revista Iberoamericana para la Investigación y el Desarrollo Educativo ISSN 2007 - 7467}

\section{Referencias}

Alfie, G. y Veloso, C. (2011). Computación práctica para docentes. México: Alfaomega.

Briceño, J. J. (2013) La argumentación y la reflexión en los procesos de mejora de los profesores universitarios colombianos de ciencia en activo. Aplicación de estrategias formativas sobre ciencia, aprendizaje y enseñanza. (tesis doctoral) Universidad de Granada, España.

Cabello, M. (2007). Sistemas de información y gestión de la empresa. Digital Learning. Recuperado de https://www.digitallearning.es/tutoriales/introduccion-a-los-sistemasde-informacion-y-gestion-de-la-empresa.html.

Camacho, P. X. (2015). Metodología Pacie como diseño instruccional para entornos virtuales de aprendizaje. Recuperado de https://es.calameo.com/read/005902376ae4826f9d6b3.

Chase, R. B. y Aquilano, N. J. (1995). Dirección y administración de la producción y de las operaciones (6. ${ }^{\mathrm{a}}$ ed.). México: Editorial Irwin.

Henríquez, M. A. (2002). La incorporación de las Tecnologías de la Información y la Comunicación en la formación inicial docente. Caso Universidad de los AndesTáchira. Revista Acción, 5(1) 60-73.

Lugo, M., López, N. y Toranzos L. (2014). Informe sobre tendencias sociales y educativas en América Latina 2014. Francia: Unesco.

Muñoz, J. L., Rodríguez, D. y Barrera, A. (2013). Herramientas para la mejora de las organizaciones educativas y su relación con el entorno. Perspectiva Educacional, 52(1), 97-123. Recuperado de http://www.perspectivaeducacional.cl/index.php/peducacional/article/view/148/0.

Osorio, M. (coord. $\left.{ }^{a}\right)$ (2015). II Congreso Internacional de Transformación Educativa. Alternativas para nuevas prácticas educativas. 3 Las tecnologías de la información y la comunicación (TIC). Ciudad de México, México: Amapsi Editorial.

Osorio, M. y Zárate, L. G. (coord. ${ }^{\text {as }}$ ) (2015). II Congreso Internacional de Transformación Educativa. Alternativas para nuevas prácticas educativas. 1 Estrategias para la transformación de la enseñanza de la lectura. Ciudad de México, México: Amapsi Editorial. 




\section{Revista Iberoamericana para la Investigación y el Desarrollo Educativo ISSN 2007 - 7467}

Polanco, I. (2008). Las tecnologías de la información y la comunicación (TIC) en la educación: retos y posibilidades. Fundación Santillana. Recuperado de http://www.fundacionsantillana.com/PDFs/xxii_semana_monografica.pdf.

Reyes, D. A. (2016) Tecnologías de información y comunicación en las organizaciones. México: Publicaciones Empresariales UNAM.

Sandoval, C. (14 de marzo de 2017). Uso de tecnología para el Aprendizaje Adaptativo. ELearning Masters.

Recuperado

de http://elearningmasters.galileo.edu/2017/03/14/tecnologia-en-el-aprendizajeadaptativo/

Sandoval, C. (29 de marzo de 2017). ¿Qué habilidades digitales deberían tener los docentes? E-Learning Masters. Recuperado de http://elearningmasters.galileo.edu/2017/03/29/habilidades-digitales-docentes/.

Silva, R., Cruz, E., Méndez, I. y Hernández, J. Á. (2013). Sistema de Gestión Digital para mejorar los procesos administrativos de Instituciones de Educación Superior: Caso de estudio en la Universidad Autónoma Metropolitana. Perspectiva Educacional, Formación de Profesores, 52(29), 104-134.

Snell, N. (1995). Internet ¿Qué hay que saber? Madrid, España: Prentice Hall..

Tapscott, D. (1997). Creciendo en un entorno digital. Bogotá, Colombia: McGraw-Hill.

Vittadini, N. (1995). Las nuevas tecnologías de comunicación. Barcelona, España: Paidós.

\section{Marina Cecilia Pérez Castillo}

Maestra en Educación Basada en Competencias por la Universidad Valle de México (UVM), Campus Veracruz, y licenciada en Contador Público y Auditor por la Universidad Cristóbal Colón. Ha cursado el diplomado en Competencias Docentes Básicas en el Centro Interdisciplinario de Investigación y Docencia en Educación Técnica (Ciidet), el diplomado en Administración Gerencial en la Universidad Veracruzana (UV) y el diplomado para la Formación y Desarrollo de Competencias Docentes. Es catedrática de tiempo completo en el Tecnológico Nacional de México, Campus Instituto Tecnológico de Veracruz. Forma parte del cuerpo académico en formación "Administración y Gestión Empresarial para el Desarrollo Humano Integral Promep”. 



Revista Iberoamericana para la
Investigación y el Desarrollo Educativo
ISSN $2007-7467$

\section{Perfecto Gabriel Trujillo Castro}

Doctor en Ciencias Jurídicas, Administrativas y de la Educación por la Universidad de las Naciones, maestro en Administración por la Universidad Cristóbal Colón e ingeniero Industrial en Producción por el Instituto Tecnológico de Veracruz. Cuenta con el diploma de Suficiencia Investigadora del Doctorado en Ciencias Económicas y Administrativas por la Universidad de Almería (España) y la Universidad de Xalapa. Tiene experiencia profesional en la industria alimenticia y naval, desarrollo rural y en instituciones educativas. Es instructor certificado de empresas: imparte cursos y conferencias y proporciona consultoría en calidad y recursos humanos. Es profesor con Perfil Deseable del Programa de Mejoramiento del Profesorado (Promep); labora en el Tecnológico Nacional de México, Campus Instituto Tecnológico de Veracruz. Es líder del cuerpo académico en formación “Administración y Gestión Empresarial para el Desarrollo Humano Integral Promep”. Ha publicado un libro y varios artículos.

\section{Sonia Báez Lagunes}

Doctora en Ciencias Jurídicas, Administrativas y de la Educación por la Universidad de las Naciones, maestra en Ciencias de la Administración por la misma universidad y licenciada en Administración de Empresas por la Universidad Veracruzana (UV). Es catedrática de tiempo completo en el Tecnológico Nacional de México, Campus Instituto Tecnológico de Veracruz, y actualmente funge como presidenta de academia de Ciencias Económico Administrativas. Cuenta con Perfil Deseable del Programa de Mejoramiento del Profesorado (Promep). Forma parte del cuerpo académico en formación “Administración y Gestión Empresarial para el Desarrollo Humano Integral Promep".

\section{Bertha Conde Carreño}

Candidata al grado en maestría en Administración Especialidad en Finanzas por la Universidad La Salle, Campus Veracruz, y licenciada en Contador Público y Auditor por la Universidad Veracruzana (UV). Es catedrática de tiempo completo en el Tecnológico Nacional de México, Campus Instituto Tecnológico de Veracruz. Forma parte del cuerpo académico en formación “Administración y Gestión Empresarial para el Desarrollo Humano Integral Promep". Y es líder de la línea de investigación en Agronegocios, coordinadora y 
colaboradora en Proyectos Integradores en la Licenciatura en Administración y actualmente secretaria de academia de Ciencias Económico Administrativas.

\section{Selene Magdala Ramos Cortés}

Candidata al grado de maestría de Impuestos por la Universidad Cristóbal Colón y licenciada en Contaduría Pública por la misma universidad. Cuenta con un diplomado en Ofimática Aplicada por el Instituto Tecnológico de Veracruz. Es consultora certificada de competencia laboral y catedrática de tiempo completo en el Tecnológico Nacional de México, Campus Instituto Tecnológico de Veracruz; imparte materias del Departamento Ciencias Económico Administrativas. Es colaboradora de Proyectos Integradores en la Licenciatura en Administración y del cuerpo académico en formación "Administración y Gestión Empresarial para el Desarrollo Humano Integral Promep". 


\begin{tabular}{|c|c|}
\hline Rol de Contribución & Autor (es) \\
\hline Conceptualización & $\begin{array}{l}\text { Marina Cecilia Pérez Castillo, principal; Perfecto Gabriel } \\
\text { Trujillo Castro, igual. }\end{array}$ \\
\hline Metodología & Perfecto Gabriel Trujillo Castro. \\
\hline Software & $\begin{array}{l}\text { Bertha Conde Carreño, principal: Selene Magdala Ramos } \\
\text { Cortés, igual }\end{array}$ \\
\hline Validación & $\begin{array}{l}\text { Perfecto Gabriel Trujillo Castro, principal; Sonia Báez } \\
\text { Lagunes, igual. }\end{array}$ \\
\hline Análisis Formal & $\begin{array}{l}\text { Sonia Báez Lagunes, principal; Bertha Conde Carreño, igual; } \\
\text { Perfecto Gabriel Trujillo Castro; igual. }\end{array}$ \\
\hline Investigación & $\begin{array}{l}\text { Marina Cecilia Pérez Castillo, principal; Perfecto Gabriel } \\
\text { Trujillo Castro; igual. }\end{array}$ \\
\hline Recursos & $\begin{array}{l}\text { Sonia Báez Lagunes, principal; Perfecto Gabriel Trujillo } \\
\text { Castro, que apoya; Bertha Conde Carreño, que apoya; Selene } \\
\text { Magdala Ramos Cortés, que apoya }\end{array}$ \\
\hline Curación de datos & $\begin{array}{l}\text { Perfecto Gabriel Trujillo Castro, principal; Sonia Báez } \\
\text { Lagunes, igual. }\end{array}$ \\
\hline $\begin{array}{l}\text { Escritura - Preparación del } \\
\text { borrador original }\end{array}$ & $\begin{array}{l}\text { Marina Cecilia Pérez Castillo, principal; Perfecto Gabriel } \\
\text { Trujillo Castro, igual. }\end{array}$ \\
\hline $\begin{array}{l}\text { Escritura - Revisión y } \\
\text { edición }\end{array}$ & $\begin{array}{l}\text { Bertha Conde Carreño, Perfecto Gabriel Trujillo Castro, } \\
\text { Marina Cecilia Pérez Castillo, Sonia Báez Lagunes, Selene } \\
\text { Magdala Ramos Cortés. Todos igual. }\end{array}$ \\
\hline Visualización & $\begin{array}{l}\text { Sonia Báez Lagunes, principal; Perfecto Gabriel Trujillo } \\
\text { Castro, igual }\end{array}$ \\
\hline Supervisión & Sonia Báez Lagunes. \\
\hline $\begin{array}{l}\text { Administración de } \\
\text { Proyectos }\end{array}$ & Perfecto Gabriel Trujillo Castro. \\
\hline Adquisición de fondos & $\begin{array}{l}\text { Bertha Conde Carreño, Perfecto Gabriel Trujillo Castro, } \\
\text { Marina Cecilia Pérez Castillo, Sonia Báez Lagunes, Selene } \\
\text { Magdala Ramos Cortés; todos Igual. }\end{array}$ \\
\hline
\end{tabular}

